\title{
Epilepsy in Tuberous Sclerosis: Phenotypes, Mechanisms, and Treatments
}

\author{
Anurag Saxena, MD (Pediatrics), MRCPCH ${ }^{1}$ Julian R. Sampson, DM, FRCP, FMedSci ${ }^{1}$ \\ 1 Division of Cancer and Genetics, Cardiff University School of \\ Medicine, Institute of Medical Genetics, Cardiff, United Kingdom \\ Semin Neurol 2015;35:269-276. \\ Address for correspondence Anurag Saxena, MD (Pediatrics), \\ MRCPCH, Institute of Medical Genetics, Cardiff University School of \\ Medicine, Heath Park, Cardiff CF14 4XN, United Kingdom \\ (e-mail: SaxenaA@cardiff.ac.uk).
}

\begin{abstract}
Keywords

- tuberous sclerosis

- TSC1

- TSC2

- mTORC1

- epilepsy

Epilepsy affects $75 \%$ to $90 \%$ of people with tuberous sclerosis, a multisystem genetic disorder. Although seizures can occur for the first time at any age, onset in infancy or childhood is usual. Around $30 \%$ of patients present with infantile spasms that often respond well to treatment with vigabatrin. Later seizures may occur as specific patterns, such as in Lennox-Gastaut syndrome, or with combinations of seizures including focal and multifocal seizures, and drop attacks. Most patients have two or more seizure types. Seizure control using current antiepileptic drugs is often unsatisfactory, leading to frequent polypharmacy. Epilepsy surgery has a place in the management of some patients. Mutations in the TSC1 and TSC2 genes that cause tuberous sclerosis lead to hyperactivation of signaling via the mammalian target of rapamycin complex 1 (mTORC1). Inhibitors of mTORC1 have recently been shown to be effective treatments for some manifestations of tuberous sclerosis; they are now being assessed as potential novel antiepileptic drugs in tuberous sclerosis and related disorders.
\end{abstract}

\section{Tuberous Sclerosis: A Multisystem Disorder}

Tuberous sclerosis, also known as tuberous sclerosis complex (TSC) is a genetic disorder affecting up to 1 in every 6,000 people. ${ }^{1}$ It is caused by mutations in the TSC1 or TSC 2 gene $^{2,3}$ and is characterized by the development of hamartomatous developmental lesions or growths in many organs. Its clinical manifestations vary between individuals both in terms of the combinations of organs involved and the severity of that involvement. Manifestations are more severe, on average, in TSC2associated than in TSC1-associated disease. ${ }^{4-7}$ The emergence of manifestations in different organs is related to age, with some aspects of brain and heart involvement becoming apparent during prenatal development; most manifestations in the skin, kidney, and lung become apparent during childhood or adult life. The diagnosis of tuberous sclerosis can be made according to clinical criteria or by genetic testing ( - Table 1$)^{8}$

Skin signs include hypomelanotic macules and fibrous plaques that often manifest in infancy, facial angiofibromas, and shagreen patches that usually develop later in childhood and periungal fibromas that typically develop in older chil- dren and adults. ${ }^{8}$ Renal involvement is mainly with angiomyolipomas that are usually multiple and bilateral. They can enlarge, particularly during adolescence and early adulthood or later, and are associated with hemorrhage, pain, and compromised renal function. ${ }^{9}$ Around $5 \%$ of patients have an early-onset form of polycystic kidney disease due to contiguous deletion of TSC2 and the adjacent PKD1 gene, which when mutated independently, causes autosomal dominant polycystic kidney disease type $1 .{ }^{10}$ Cardiac rhabdomyomas occur in more than half of infants with TSC. They are often multiple and can be diagnosed by antenatal ultrasound scan. Spontaneous postnatal resolution of cardiac rhabdomyomas is usual, in contrast to other manifestations in TSC. ${ }^{11}$ Lung involvement with lymphangioleiomyomatosis is usually only clinically significant in adult women, and presents with cough, hemoptysis, shortness of breath, or pneumothorax, and may progress to respiratory failure. ${ }^{12}$

The central nervous system is involved in virtually all affected individuals and leads to problems including epilepsy, cognitive deficits, autism, and other neurodevelopmental and behavioral disorders, anxiety and depression. These problems
Issue Theme Etiology of Epilepsy; Guest Editors: Philip Smith, MD, FRCP,

FAcadMEd, and Rhys Thomas, BSc, MRCP, MSc, PhD
Copyright $\odot 2015$ by Thieme Medical Publishers, Inc., 333 Seventh Avenue, New York, NY 10001, USA. Tel: +1(212) 584-4662.
DOI http://dx.doi.org/ 10.1055/s-0035-1552616. ISSN 0271-8235. 
Table 1 Diagnostic criteria for tuberous sclerosis, updated $2012^{8}$

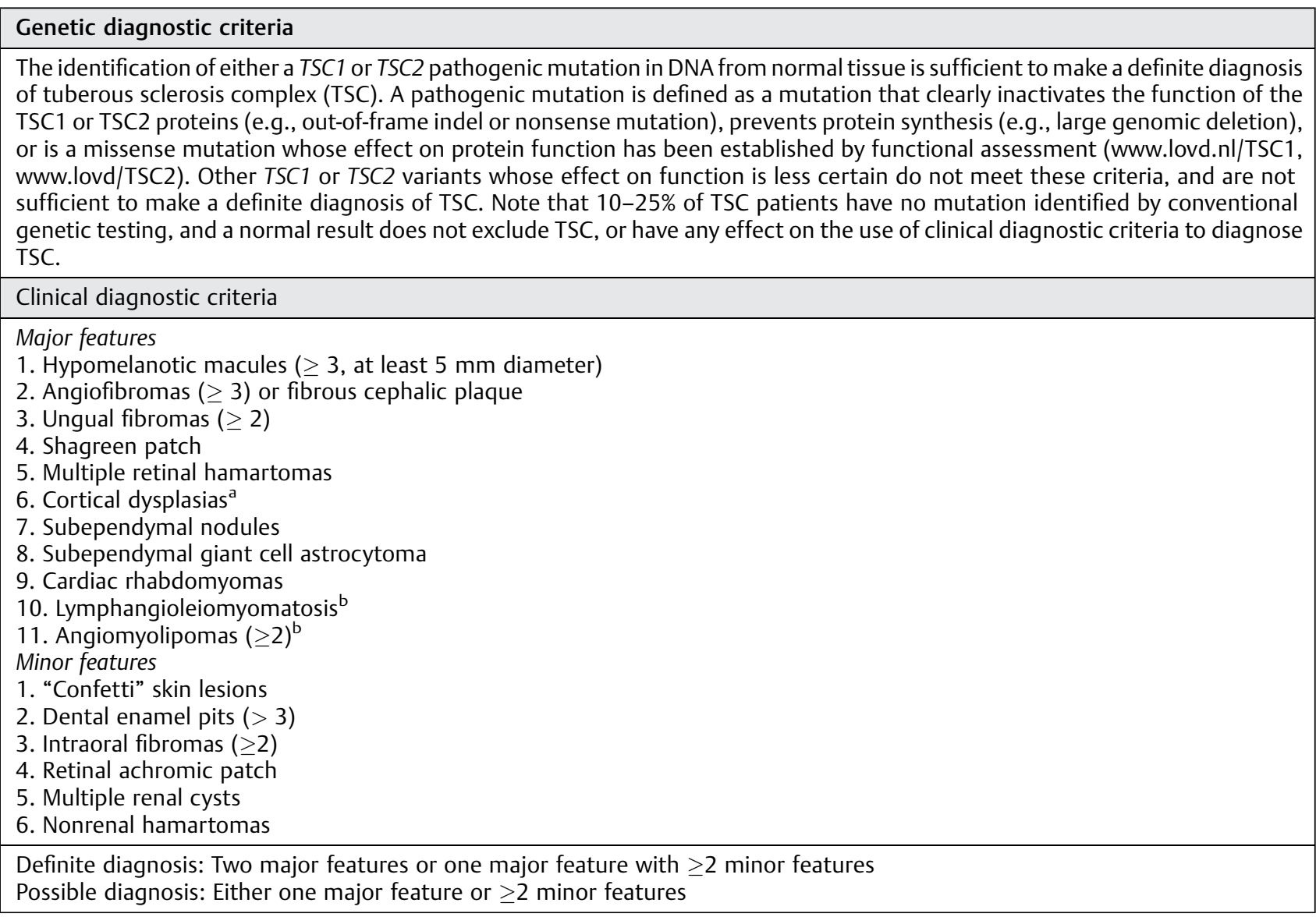

ancludes tubers and cerebral white matter radial migration lines.

${ }^{\mathrm{b}} \mathrm{A}$ combination of the two major clinical features (lymphangioleiomyomatosis and angiomyolipomas) without other features does not meet criteria for a definite diagnosis.

are rated by patients and their families as the most important consequences of the condition. ${ }^{13}$ Epilepsy is the most common symptom of brain involvement and develops in $75 \%$ to $90 \%$ of people with TSC. ${ }^{14,15}$ Psychiatric problems are underrecognized, and a checklist of TSC-associated neuropsychiatric disorders has been developed recently to improve their detection in the clinic. ${ }^{16,17}$

The structural hallmarks of CNS involvement are developmental abnormalities including cortical tubers, subependymal nodules, heterotopic gray and white matter abnormalities ${ }^{18-21}$ (-Fig. 1), and brain tumors, termed subependymal giant cell astrocytomas (SEGAs), that typically develop in childhood or early adult life. ${ }^{1}$ White matter changes on standard magnetic resonance imaging (MRI) feature curvilinear radial bands extending from the ventricles toward the cortex, wedgeshaped lesions, nonspecific focal conglomerate changes, and cerebellar radial bands. ${ }^{22}$ Diffusion tensor imaging (DTI), a structural MRI technique used to examine the interconnections between different brain regions, is more sensitive than standard MRI in identifying microstructural abnormalities in brain pathways. Recent DTI studies in TSC have shown widespread disorganization in otherwise normal-appearing white matter $^{23,24}$ and changes in the frontal and parietal lobes that appear to correlate with attention deficits. ${ }^{25}$

\section{TSC1, TSC2, and Signaling via mTORC1}

The proteins encoded by TSC1 and TSC2 (referred to as TSC1 and TSC2 or hamartin and tuberin, respectively) form a complex with TBC1D7 within cells that acts as a negative regulator of mammalian (or mechanistic) target of rapamycin complex 1 (mTORC1). ${ }^{26}$ mTORC1 is activated by amino acids, growth factors, and energy status (AMP/ATP ratio); its best characterized downstream targets include ribosomal protein S6 kinase 1 (S6K1) and eukaryotic initiation factor 4E-binding

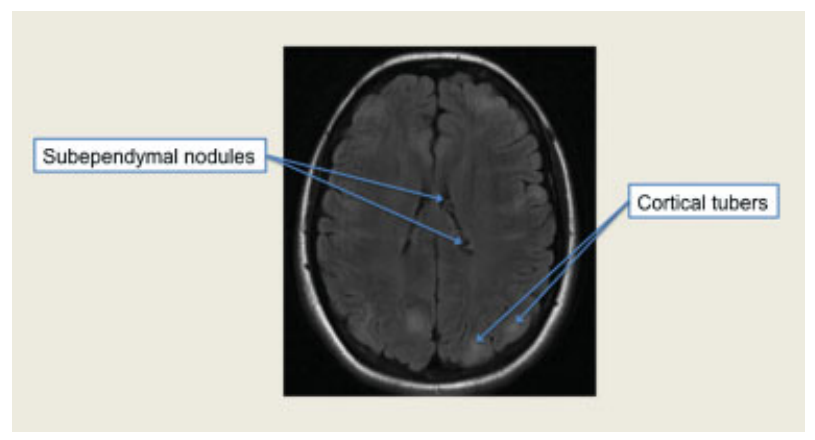

Fig. 1 Magnetic resonance image of the brain (axial T1-weighted image) of a patient with tuberous sclerosis complex showing typical findings of cortical tubers and subependymal nodules. 
protein 1 (4E-BP1), through which protein synthesis is regulated. Other processes regulated by mTORC1 include lipogenesis, angiogenesis, glycolysis, autophagy, inflammatory responses and neuronal migration, differentiation, and function. ${ }^{27-33}$ Loss of functional TSC1/2 leads to overactivation of mTORC1 and perturbation of developmental pathways, the balance of anabolic and catabolic processes and physiological responses to variation in external conditions. Recent research has shown that many (if not all) of the major manifestations of TSC result from overactivation of mTORC1 signaling. The mTORC1 signaling pathway is also dysregulated in diverse disease states, including neurodegeration, diabetes mellitus, epilepsy, and cancer. Inherited mutations in other components of the signaling pathway cause genetic disorders that are characterized by various combinations of tumor predisposition, cerebral cortical dysplasia, epilepsy, and neurodevelopmental problems. ${ }^{34}$

TSC1 and TSC2 act as tumor suppressors and somatic "second hit" mutations occur in many TSC-associated hamartomas. $^{35,36}$ In the brain, the role of second hit mutations in determining in utero development of cortical tubers is less clear, probably reflecting cellular heterogeneity of these lesions. Nonetheless, there is evidence of upregulated mTORC1 activity in cortical tubers from both adult ${ }^{37}$ and fetal brains. ${ }^{18,19}$ Preclinical studies using mouse models suggest that mTORC1 inhibition may normalize aspects of brain development in the context of TSC deficiency, ${ }^{19}$ further supporting mTORC1 activation as critical to pathogenesis of structural brain abnormalities in TSC.

\section{Multiple Epilepsy Phenotypes in Infants, Children, and Adults with TSC}

Approximately $30 \%$ of TSC cases present with infantile spasms, the hallmark seizure type of West syndrome, an epileptic encephalopathy syndrome of infancy. Some go on to develop a clinical picture of Lennox-Gastaut syndrome with persisting severe epilepsy. ${ }^{38}$ In children and adults with TSC, the typical seizure types are focal and focal dyscognitive seizures, but many also have other seizure semiology such as tonic, clonic, tonic-clonic, myoclonic, atonic, and atypical absences. ${ }^{15}$ Unfavorable prognostic factors for epilepsy in TSC include seizure onset in the first year of life, the presence of multiple seizure types, multifocal electroencephalogram (EEG) discharges when awake that generalize in sleep and/or secondary bilateral synchrony, and the onset of new EEG foci during evolution of epilepsy. ${ }^{39}$ TSC2 mutations are associated with a significantly earlier presentation of epilepsy than TSC1 mutations, a higher likelihood of infantile spasms, and a worse outcome with regards to seizure control and neurodevelopment. ${ }^{4}$

\section{Infantile Spasms in Tuberous Sclerosis Complex}

Tuberous sclerosis complex is the most common single cause of infantile spasms, accounting for up to $25 \%$ of all cases. ${ }^{15}$ Their onset in TSC peaks at 4 to 6 months of age, in common with infantile spams from other causes. Infantile spasms are characterized by a triad of developmental arrest and/or regression, typical epileptic spasms, and a high-voltage chaotic EEG pattern known as hypsarrhythmia. Irritability may precede the onset of spasms; the affected infant often shows behavioral regression manifesting as indifference to his or her environment and parents, ${ }^{40}$ together with cessation or regression of wider developmental abilities. The spasms are usually characterized by bilateral, tonic contraction of muscles in the neck, trunk, and extremities, which may be flexor and/or extensor. The contraction appears to have two phases with an intense, quick phase lasting for a couple of seconds, followed by a longer period of motor arrest during which the posture acquired in the preceding phase is maintained. Some infants may present in a very subtle fashion with head nods, elevation of the shoulders or upward eye deviation, and crying that may be confused with colic.

The spasms typically occur as clusters, and are most likely to be noticed first at the sleep-wake interface. Clusters of spasms may occur a few times per day or up to 100 or more, 5 to 30 seconds apart. There may be many such clusters during a day, and they may be followed by irritability, lethargy, or occasionally an apparent state of hyperalertness.

Although infantile spams in TSC are clinically similar to those due to other causes, there are differences. In TSC the spasms may present very early ${ }^{15}$ and be preceded by, or coexist with, focal seizures. Subtle early focal seizures, such as unilateral tonic or clonic phenomena affecting the face or limbs, tonic eye deviation, head turning, and unilateral grimacing may go unrecognized until there are more obvious infantile spasms. $^{38,41}$

\section{Electroencephalogram Findings}

Hypsarrhythmia (hypselos-Greek for "high") is the typical electrophysiological picture in infantile spasms. It is an interictal EEG pattern defined as a diffuse, high voltage ( $>$ $200 \mu \mathrm{V})$, irregular, chaotic mixture of slow waves with sharp waves and spikes. The localization of sharp waves and spikes typically shifts temporally, although in TSC infants it may differ in having an additional consistent focus. This EEG pattern occurs both when awake and in non-rapid-eyemovement (non-REM) sleep, with marked attenuation of the pattern in REM sleep. The typical features of hypsarrhythmia may only be evident during sleep in the initial phase of infantile spasms, making a sleep EEG recording essential. The ictal phase (associated with an epileptic spasm) typically has an initial positive wave over the vertex-central region that evolves into a period of abrupt generalized desynchronization (flattening) called the "electro-decremental response," lasting for several seconds. ${ }^{42}$

\section{Management of Infantile Spasms}

Vigabatrin is very effective in managing infantile spasms due to TSC and is the drug of choice in this situation. ${ }^{6}$ If vigabatrin is unavailable or is ineffective, other treatments such as corticosteroids, sodium valproate, topiramate, or zonisamide should be tried. The imperative is to control the seizures quickly. Vigabatrin carries the potentially serious side effect 
of retinal toxicity and permanent peripheral visual field constriction (www.tsalliance.org/documents/VigabatrinAssociatedVisualFieldLossWhitePaper.pdf). This affects some $30 \%$ of treated of adults, but the risk in children is probably lower. ${ }^{43}$ The prevalence of clinically significant visual problems attributable to vigabatrin is much lower, probably $1 \%$ to $5 \%$. We do not know either the best surveillance method or the extent to which toxicity may be reversible on stopping treatment. The recommended regular kinetic perimetry for visual field testing is not an option for infants; electroretinogram or optical coherence tomography should be considered. The potential consequences of vigabatrin for vision need to be balanced with the neurodevelopmental consequences of not treating infantile spasms effectively.

\section{Infantile Spasms and Neurodevelopmental Outcome}

Infantile spasms in TSC increase the risk of subsequent intellectual difficulties, autism or autism-spectrum disorder, behavioral problems, and epilepsy that is difficult to control. ${ }^{44-47}$ However, several studies have shown that some children with TSC and infantile spasms have normal cognitive outcomes, particularly if seizure control is achieved early. ${ }^{48-51}$ Vigabatrin effectively controls infantile spasms in up to $96 \%$ of TSC cases and appears to have a positive impact on neurocognitive outcome. ${ }^{52}$ EPISTOP is an ongoing pioneering study in infants with TSC and the first prospective study of epileptogenesis in humans. The trial monitors TSC babies using serial EEGs. Clinicians may use antiepileptic medication (usually vigabatrin) on the basis of a deteriorating EEG before the onset of clinical seizures. The trial will assess the impact on neurodevelopmental outcome over 2 years or more (clinicaltrials.gov identifier NCT02098759).

\section{Lennox-Gastaut Syndrome}

Most children developing Lennox-Gastaut syndrome have had prior epilepsy, commonly infantile spasms. The triad of symptoms that develop aged 1 to 8 years are (1) seizures including a mix of myoclonic seizures, atonic, axial tonic, atypical absences, generalized tonic-clonic and focal seizures; (2) an EEG featuring diffuse slow, spike-wave and burst of fast activity during sleep; and (3) learning difficulties and behavioral problems. ${ }^{15,42}$ Most children go on to have refractory epilepsy as adults and are at risk of poor neurocognitive outcomes.

\section{Electroencephalogram Findings}

The diagnostic feature is multifocal interictal 2.0 to $2.5 \mathrm{~Hz}$ sharp-and-slow wave discharges. Eye opening or photic stimulation do not affect the discharges. The EEG can be activated during sleep, especially in stage 1,2, and REM sleep, and may sometimes be activated by hyperventilation. ${ }^{42}$

The ictal EEG changes depend on the seizure semiology. Tonic seizures are associated with a burst of bilateral rhythmic 10 to $20 \mathrm{~Hz}$ activity over anterior areas and the vertex. Atypical absences on the other hand are associated with sharp-and-slow wave discharges, indistinguishable from the interictal phase. Electroencephalogram changes with atonic and atonic-myoclonic seizures show either multiple or diffuse spike-and-slow activity or fast rhythms with an anterior predominance. ${ }^{42}$

\section{Treatment and Outcome}

A recent Cochrane review concluded that the optimum treatment for Lennox-Gastaut syndrome remains uncertain, and no study to date has shown any one drug to be highly effective. ${ }^{53}$ Rufinamide, lamotrigine, topiramate, and felbamate may be considered as add-on therapies, and clobazam may help for drop seizures. However, total seizure control is rarely achieved.

\section{Epilepsy Associated with TSC in Older Children and Adults}

Epilepsy in children and adults with TSC has an unpredictable course. Patients show multiple seizure types, most likely reflecting the presence of multiple tubers with variable epileptogenic potential in most patients. More than half of TSC adults have more than one seizure type, with focal onset seizures in most (93\%). ${ }^{54}$ At least one third of epilepsy in older children and adults is refractory, despite using multiple antiepileptic medications and nonpharmacological approaches. ${ }^{55-58}$ In comparison to TSC patients with epilepsy who are controlled on medication, the refractory group is characterized by a younger age at diagnosis, a previous history of infantile spasms and/or Lennox-Gastaut syndrome, lower educational achievement, a higher prevalence of psychiatric problems, and association with TSC2 mutations. ${ }^{57}$

\section{Antiepileptic Drugs}

Epilepsy management is best coordinated through a multidisciplinary clinic with specialist expertise in epilepsy because of the frequent association of epilepsy with intellectual difficulties, neurodevelopmental and behavioral problems in patients with TSC, and the frequency of TSC-associated comorbidities, particularly kidney disease. ${ }^{59}$

Carbamazepine for focal-onset epilepsy and sodium valproate for generalized and myoclonic seizures are frequently used first-line therapies. Seizure control by monotherapy is relatively infrequent, and combination therapy with medications, including lamotrigine, topiramate, levetiracetam, oxcarbazepine, rufinamide, clobazam, and clonazepam, is often required. The combination of levetiracetam with antiepileptic drugs that enhance GABAergic inhibition, or the combination of sodium valproate and lamotrigine, may be considered. ${ }^{60,61}$ Complicated combinations of antiepileptic drugs rarely provide an additional benefit and can leave a high burden of side effects. It is therefore important to define realistic treatment expectations in "hard-to-treat" patients. ${ }^{58}$

The refractory nature of epilepsy in TSC likely reflects underlying multifocal cortical dysgenesis and perhaps the wider consequences of dysregulated mTORC1 signaling in the brain. The proteins MDR-1 (multidrug resistance) and MRP-1 (multidrug resistance-associated protein-1) are linked to chemotherapy resistance in tumor cells. The presence of these 
proteins in cortical tubers suggests they may also play a role in development of refractory epilepsy. ${ }^{62}$ Persisting severe epilepsy in the face of expert management with antiepileptic drugs should prompt consideration of nonpharmacological management, including epilepsy surgery, vagus nerve stimulation, or ketogenic diet.

\section{Epilepsy Surgery}

\section{Resective Epilepsy Surgery}

It is worth considering resective surgery for patients with refractory epilepsy who have an identifiable epileptogenic zone associated with one or more glioneuronal hamartomas, ideally in noneloquent cortex, even in those (the majority) with multiple cortical tubers. ${ }^{63}$ With expert assessment and selection of patients, most cases achieve a meaningful longterm reduction of seizure burden and use less antiepileptic therapy; some cases achieve complete remission. ${ }^{64}$

\section{Corpus Callosotomy}

Corpus callosotomy may be considered in children who have drop attacks as their most disabling seizure type. ${ }^{65}$ Drop attacks (atonic seizures) can occur many times every day and lead to serious injuries. The possibility of inducing a disconnection syndrome through complete callosotomy must be weighed against the dangers and quality-of-life issues posed

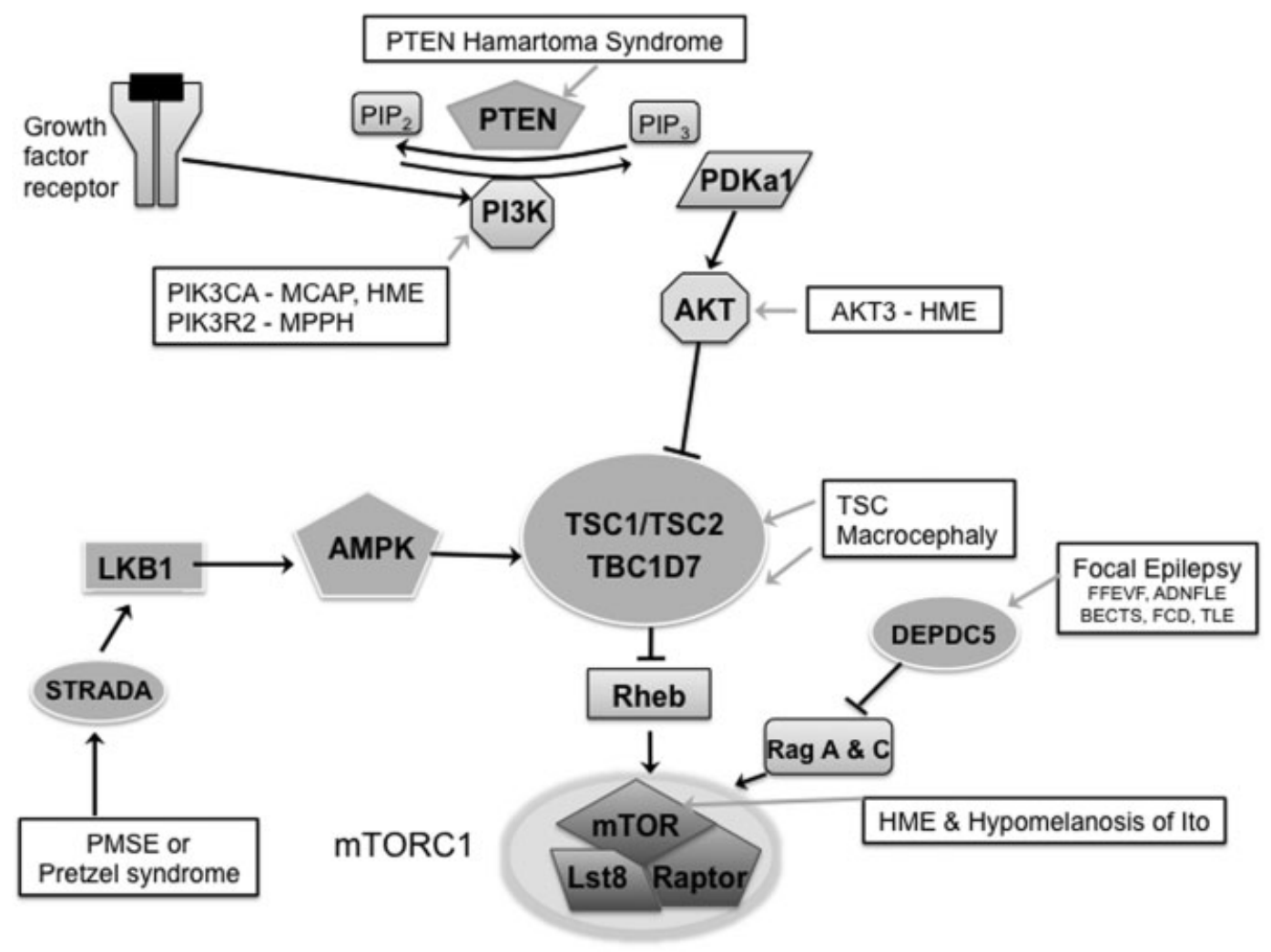

Fig. 2 Simplified representation of the signaling pathway upstream of mTORC1 showing disorders associated with mutations affecting the genes encoding pathway components. mTORC1 is composed of mTOR, regulatory-associated protein of mTOR (Raptor) and mammalian lethal with SEC13 protein 8 (LST8). Negative regulators of mTORC1 are shaded dark gray; positive regulators are shaded light gray. Disorders related to epilepsy are indicated in white boxes. Key upstream components in the mTOR signaling pathway include PI3K (phosphoinositide 3-kinase), PTEN (phosphatase and tensin homolog), PDK1 (phosphoinositide dependent protein kinase), AKT ( $v$-akt murine thymoma viral oncogene), AMPK (AMP-dependent protein kinase), TSC1 and 2 (tuberous sclerosis complex $1 \& 2$ ). The TSC1/2 protein complex is a major negative regulator of mTORC1 activity. Its function is mediated via TSC2's inhibition of Rheb. TBC1D7 (TBC1 domain family, member 7) is a third subunit of the TSC1/2 complex. Homozygous mutations in TBC1D7 are associated with macrocephaly, intellectual disability and neuropsychological disorders. PTEN converts the PIP3 (phosphatidylinositol 3,4,5 triphosphate) to PIP2 (phosphatidylinositol 4,5 bisphosphate), thereby reducing activity of PI3K, PDK1 and AKT, which leads to mTORC1 inhibition. A reduction in PTEN activity therefore leads to mTORC1 activation. Mutations of PIK3CA have been identified in patients with megalencephaly-capillary malformation syndrome (MCAP) and hemimegalencephaly (HME), whereas activating mutations of PIK3R2 or AKT3 have been identified in most patients with megalencephaly-polymicrogyria-polydactyly-hydrocephalus syndrome $(\mathrm{MPPH})$ and some with hemimegalencephaly (HME). Truncating mutations in DEPDC5 (disheveled, Egl-10 and pleckstrin domain containing protein 5) have been described in patients with a broad range of focal epilepsy phenotypes: familial focal epilepsy with variable foci (FFEVF), autosomal dominant nocturnal frontal lobe epilepsy (ADNFLE), familial temporal lobe epilepsy (TLE), benign epilepsy of childhood with centrotemporal spikes (BECTS or Rolandic epilepsy) and cases with focal cortical dysplasia (FCD) mediated through the RAS-related GTP-binding protein (RAG) proteins A and C. PMSE syndrome (polyhydramnios, megalencephaly, and symptomatic epilepsy), also known as Pretzel syndrome, results from mutations in STRADA (STE20-related kinase adaptor $\alpha$ ) that prevents its activation and binding with LKB1 (liver kinase B), resulting in reduction in AMPK- (AMP-dependent protein kinase) mediated TSC2 activation and hence mTORC1 activation. ( Adapted from Saxena A, Sampson JR. Phenotypes associated with inherited and developmental somatic mutations in genes encoding mTOR pathway components. Sem Cell Developm Biol 2014;36:140-146.) 
by the seizures. Focal seizures usually persist after callosotomy; such patients may need additional antiepileptic drug treatment and/or secondary surgery to resect the epileptogenic tuber or tubers. ${ }^{66}$

\section{Vagus Nerve Stimulation}

Vagus nerve stimulation is used as an adjunctive therapy for intractable focal-onset seizures in patients aged over 12 years. The antiepileptic effects of vagus nerve stimulation are mediated by altered vagal afferent activities, and probably include altered activities in the reticular activating system, the central autonomic network, the limbic system, and the diffuse noradrenergic projection system. ${ }^{67}$ Common side effects include cough, hoarseness, voice alteration, and throat paresthesias. It is uncertain whether the efficacy of vagus nerve stimulation in TSC is comparable to other cases with refractory seizures, where there are reported median seizure reductions compared with baseline of $35 \%$ at 1 year to $44 \%$ at 3 years. ${ }^{68}$ Studies reporting efficacy of vagus nerve stimulation in TSC-related epilepsy have been small, but suggest that results may be comparable to those achieved in other settings. ${ }^{56,69,70}$

\section{Ketogenic Diet}

A ketogenic diet may be used independently or combined with other treatment modalities to manage difficult-to-treat seizures. It is a high fat, low carbohydrate, and adequate protein diet that results in ketosis; this may exert its antiepileptic effects via mechanisms including enhanced GABA levels, reduced neuronal excitability and firing, stabilization of synaptic function, and inhibition of the mTOR pathway and glutaminergic excitatory synaptic transmission. ${ }^{71,72} \mathrm{~A}$ recent Cochrane review of the ketogenic diet in diverse intractable epilepsies in childhood showed short-to-medium term benefits in seizure control comparable to antiepileptic drugs, but poor long-term compliance. ${ }^{73}$ Studies in adults are very limited.

\section{Pathophysiology of Epilepsy in TSC: Evidence for the Role of mTORC1 Hyperactivation}

In conditional transgenic mouse models, homozygous knockout of TSC1 or TSC2 in various brain cell populations led to hyperactivation of mTORC1 signaling, developmental abnormalities of the brain, seizures, and reduced lifespan. ${ }^{74,75}$ Brain pathology showed gross enlargement with ectopic, enlarged, and aberrant neurons similar to the dysplastic neurons found in the cortical tubers of TSC. Brain size and the histopathological abnormalities were reversed by treatment with the mTORC1 inhibitors rapamycin and everolimus with cessation of seizures and dramatic effects on survival. ${ }^{76}$ Elevated mTORC1 activity has also been shown in cortical tubers removed from TSC patients undergoing epilepsy surgery. ${ }^{77}$ An open-label phase I/II clinical trial suggested that mTORC1 inhibitors present new opportunities for treatment of epilepsy in TSC. $^{78,79}$ An international multicenter randomized clinical trial, EXIST3 (NCT01713946) is investigating the efficacy and safety of everolimus as an adjunctive therapy in TSC-associated epilepsy.

\section{Role of mTORC1 Hyperactivity in Other Epilepsy-Associated Disorders}

A group of related rare disorders is caused by inherited or somatic developmental mutations that affect components of the MTORC1 signaling pathway and that lead to its overactivity (-Fig. 2). ${ }^{34,80-83}$ These disorders predispose to seizures and include hemimegalencephaly, Pretzel or PMSE (polyhydramnios, megalencephaly, and symptomatic epilepsy) syndrome, megalencephaly-polymicrogyria-polydactyly-hydrocephalus (MPPH) and megalencephaly-capillary malformation (MCAP) syndromes, and the recently described DEPDC5(disheveled, Egl-10, and pleckstrin domain containing protein 5) associated epilepsies. Overactive mTORC1 activity has also been documented apparently unrelated epilepsy syndromes, such as hippocampal sclerosis and Rasmussen encephalitis. ${ }^{37}$ These observations suggest that mTORC1 inhibition holds promise for treatment of epilepsy in a diversity of clinical settings.

\section{Conclusions}

Epilepsy is an important manifestation of TSC and affects the vast majority of patients with this inherited condition. It is frequently difficult to control, requiring the use of multiple antiepileptic drugs and further measures, including epilepsy surgery in some cases. Early onset of seizures, particularly infantile spasms, is associated with a poor neurocognitive outcome, and the seizures may directly contribute to these problems. Many of the manifestations of tuberous sclerosis are at least in part directly attributable to hyperactivation of mTORC1, and preclinical and early clinical evidence suggest this may also be the case for TSC-associated epilepsy, opening up the possibility of using mTORC1 inhibitors as antiepileptic drugs in patients with TSC.

\section{Acknowledgments}

The authors' research is supported by Wales Gene Park, the Tuberous Sclerosis Association, and Novaris Pharmaceuticals.

\footnotetext{
References

1 Crino PB, Nathanson KL, Henske EP. The tuberous sclerosis complex. N Engl J Med 2006;355(13):1345-1356

2 European Chromosome 16 Tuberous Sclerosis Consortium. Identification and characterization of the tuberous sclerosis gene on chromosome 16. Cell 1993;75(7):1305-1315

3 van Slegtenhorst M, de Hoogt R, Hermans C, et al. Identification of the tuberous sclerosis gene TSC1 on chromosome 9q34. Science 1997;277(5327):805-808

4 Dabora SL, Jozwiak S, Franz DN, et al. Mutational analysis in a cohort of 224 tuberous sclerosis patients indicates increased severity of TSC2, compared with TSC1, disease in multiple organs. Am J Hum Genet 2001;68(1):64-80
} 
5 Sancak O, Nellist M, Goedbloed M, et al. Mutational analysis of the TSC1 and TSC2 genes in a diagnostic setting: genotype-phenotype correlations and comparison of diagnostic DNA techniques in Tuberous Sclerosis Complex. Eur J Hum Genet 2005;13(6): 731-741

6 Wang S, Fallah A. Optimal management of seizures associated with tuberous sclerosis complex: current and emerging options. Neuropsychiatr Dis Treat 2014;10:2021-2030

7 Lewis JC, Thomas HV, Murphy KC, Sampson JR. Genotype and psychological phenotype in tuberous sclerosis. J Med Genet 2004; 41(3):203-207

8 Northrup H, Krueger DA; International Tuberous Sclerosis Complex Consensus Group. Tuberous sclerosis complex diagnostic criteria update: recommendations of the 2012 International Tuberous Sclerosis Complex Consensus Conference. Pediatr Neurol 2013;49(4):243-254

9 Bissler JJ, Kingswood JC. Renal angiomyolipomata. Kidney Int 2004;66(3):924-934

10 Sampson JR, Maheshwar MM, Aspinwall R, et al. Renal cystic disease in tuberous sclerosis: role of the polycystic kidney disease 1 gene. Am J Hum Genet 1997;61(4):843-851

11 Bosi G, Lintermans JP, Pellegrino PA, Svaluto-Moreolo G, Vliers A. The natural history of cardiac rhabdomyoma with and without tuberous sclerosis. Acta Paediatr 1996;85(8):928-931

12 McCormack FX, Inoue Y, Moss J, et al. Efficacy and safety of sirolimus in lymphangioleiomyomatosis. N Engl J Med 2011; 364(17):1595-1606

13 Hunt A. Tuberous sclerosis: a survey of 97 cases. III: Family aspects. Dev Med Child Neurol 1983;25(3):353-357

14 Webb DW, Fryer AE, Osborne JP. On the incidence of fits and mental retardation in tuberous sclerosis. J Med Genet 1991;28(6): 395-397

15 Thiele EA. Managing epilepsy in tuberous sclerosis complex. J Child Neurol 2004;19(9):680-686

16 de Vries PJ, Whittemore VH, Leclezio L, et al. Tuberous sclerosis associated neuropsychiatric disorders (TAND) and the TAND Checklist. Pediatr Neurol 2015;52(1):25-35

17 Leclezio L, Jansen A, Whittemore VH, de Vries PJ. Pilot validation of the tuberous sclerosis-associated neuropsychiatric disorders (TAND) checklist. Pediatr Neurol 2015;52(1):16-24

18 Prabowo AS, Anink JJ, Lammens M, et al. Fetal brain lesions in tuberous sclerosis complex: TORC1 activation and inflammation. Brain Pathol 2013;23(1):45-59

19 Tsai V, Parker WE, Orlova KA, et al. Fetal brain mTOR signaling activation in tuberous sclerosis complex. Cereb Cortex 2014; 24(2):315-327

20 Park SH, Pepkowitz SH, Kerfoot C, et al. Tuberous sclerosis in a 20week gestation fetus: immunohistochemical study. Acta Neuropathol 1997;94(2):180-186

21 Luat AF, Makki M, Chugani HT. Neuroimaging in tuberous sclerosis complex. Curr Opin Neurol 2007;20(2):142-150

22 Braffman BH, Bilaniuk LT, Naidich TP, et al. MR imaging of tuberous sclerosis: pathogenesis of this phakomatosis, use of gadopentetate dimeglumine, and literature review. Radiology 1992;183(1): 227-238

23 Makki MI, Chugani DC, Janisse J, Chugani HT. Characteristics of abnormal diffusivity in normal-appearing white matter investigated with diffusion tensor MR imaging in tuberous sclerosis complex. AJNR Am J Neuroradiol 2007;28(9):1662-1667

24 Arulrajah S, Ertan G, Jordan L, et al. Magnetic resonance imaging and diffusion-weighted imaging of normal-appearing white matter in children and young adults with tuberous sclerosis complex. Neuroradiology 2009;51(11):781-786

25 Peng SS, Lee WT, Wang YH, Huang KM. Cerebral diffusion tensor images in children with tuberous sclerosis: a preliminary report. Pediatr Radiol 2004;34(5):387-392
26 Dibble CC, Elis W, Menon S, et al. TBC1D7 is a third subunit of the TSC1-TSC2 complex upstream of mTORC1. Mol Cell 2012;47(4): 535-546

27 Laplante M, Sabatini DM. Regulation of mTORC1 and its impact on gene expression at a glance. J Cell Sci 2013;126(Pt 8):1713-1719

28 Yecies JL, Manning BD. mTOR links oncogenic signaling to tumor cell metabolism. J Mol Med (Berl) 2011;89(3):221-228

29 Parkhitko AA, Favorova OO, Khabibullin DI, Anisimov VN, Henske EP. Kinase mTOR: regulation and role in maintenance of cellular homeostasis, tumor development, and aging. Biochemistry (Mosc) 2014;79(2):88-101

30 Au KS, Williams AT, Gambello MJ, Northrup H. Molecular genetic basis of tuberous sclerosis complex: from bench to bedside. J Child Neurol 2004;19(9):699-709

31 Huang J, Manning BD. The TSC1-TSC2 complex: a molecular switchboard controlling cell growth. Biochem J 2008;412(2): 179-190

32 Kim SG, Buel GR, Blenis J. Nutrient regulation of the mTOR complex 1 signaling pathway. Mol Cells 2013;35(6):463-473

33 Ehninger D, Han S, Shilyansky C, et al. Reversal of learning deficits in a Tsc2+/- mouse model of tuberous sclerosis. Nat Med 2008; 14(8):843-848

34 Saxena A, Sampson JR. Phenotypes associated with inherited and developmental somatic mutations in genes encoding mTOR pathway components. Semin Cell Dev Biol 2014;36:140-146

35 Tyburczy ME, Wang JA, Li S, et al. Sun exposure causes somatic second-hit mutations and angiofibroma development in tuberous sclerosis complex. Hum Mol Genet 2014;23(8):2023-2029

36 Carbonara C, Longa L, Grosso E, et al. Apparent preferential loss of heterozygosity at TSC2 over TSC1 chromosomal region in tuberous sclerosis hamartomas. Genes Chromosomes Cancer 1996;15(1): $18-25$

37 Liu J, Reeves C, Michalak Z, et al. Evidence for mTOR pathway activation in a spectrum of epilepsy-associated pathologies. Acta Neuropathol Commun 2014;2:71

38 Curatolo P, Verdecchia M, Bombardieri R. Tuberous sclerosis complex: a review of neurological aspects. Eur J Paediatr Neurol 2002;6(1):15-23

39 Curatolo P, Bombardieri R, Verdecchia M, Seri S. Intractable seizures in tuberous sclerosis complex: from molecular pathogenesis to the rationale for treatment. J Child Neurol 2005;20(4): 318-325

40 Guzzetta F, Crisafulli A, Isaya Crinó M. Cognitive assessment of infants with West syndrome: how useful is it for diagnosis and prognosis? Dev Med Child Neurol 1993;35(5):379-387

41 Curatolo P, Seri S, Verdecchia M, Bombardieri R. Infantile spasms in tuberous sclerosis complex. Brain Dev 2001;23(7):502-507

42 Pressler RMBC, Cooper R, Robinson R. Neonatal and Paediatric Clinical Neurophysiology. Amsterdam, The Netherlands: Elsevier; 2007

43 Kinirons P, Cavalleri GL, O'Rourke D, et al. Vigabatrin retinopathy in an Irish cohort: lack of correlation with dose. Epilepsia 2006;47(2): 311-317

44 Bolton PF, Park RJ, Higgins JN, Griffiths PD, Pickles A. Neuroepileptic determinants of autism spectrum disorders in tuberous sclerosis complex. Brain 2002;125(Pt 6):1247-1255

45 Curatolo P, D’Argenzio L, Cerminara C, Bombardieri R. Management of epilepsy in tuberous sclerosis complex. Expert Rev Neurother 2008;8(3):457-467

46 Joinson C, O'Callaghan FJ, Osborne JP, Martyn C, Harris T, Bolton PF. Learning disability and epilepsy in an epidemiological sample of individuals with tuberous sclerosis complex. Psychol Med 2003; 33(2):335-344

47 O'Callaghan FJ, Harris T, Joinson C, et al. The relation of infantile spasms, tubers, and intelligence in tuberous sclerosis complex. Arch Dis Child 2004;89(6):530-533 
48 Hamano S, Tanaka M, Mochizuki M, Sugiyama N, Eto Y. Long-term follow-up study of West syndrome: differences of outcome among symptomatic etiologies. J Pediatr 2003;143(2):231-235

49 Fukushima K, Inoue Y, Fujiwara T, Yagi K. Long-term follow-up study of West syndrome associated with tuberous sclerosis. Brain Dev 2001;23(7):698-704

50 Lane VW, Samples JM. Tuberous sclerosis: case study of early seizure control and subsequent normal development. J Autism Dev Disord 1984;14(4):423-427

51 Jambaqué I, Chiron C, Dumas C, Mumford J, Dulac O. Mental and behavioural outcome of infantile epilepsy treated by vigabatrin in tuberous sclerosis patients. Epilepsy Res 2000;38(2-3):151-160

52 Aicardi J, Mumford JP, Dumas C, Wood S; Sabril IS Investigator and Peer Review Groups. Vigabatrin as initial therapy for infantile spasms: a European retrospective survey. Epilepsia 1996;37(7): 638-642

53 Hancock EC, Cross JH. Treatment of Lennox-Gastaut syndrome. Cochrane Database Syst Rev 2013;2:CD003277

54 Chu-Shore CJ, Major P, Camposano S, Muzykewicz D, Thiele EA. The natural history of epilepsy in tuberous sclerosis complex. Epilepsia 2010;51(7):1236-1241

55 Curatolo P. Neurological manifestations of tuberous sclerosis complex. Childs Nerv Syst 1996;12(9):515-521

56 Elliott RE, Carlson C, Kalhorn SP, et al. Refractory epilepsy in tuberous sclerosis: vagus nerve stimulation with or without subsequent resective surgery. Epilepsy Behav 2009;16(3): 454-460

57 Vignoli A, La Briola F, Turner K, et al. Epilepsy in TSC: certain etiology does not mean certain prognosis. Epilepsia 2013;54(12): 2134-2142

58 Moavero R, Cerminara C, Curatolo P. Epilepsy secondary to tuberous sclerosis: lessons learned and current challenges. Childs Nerv Syst 2010;26(11):1495-1504

59 Krueger DA, Northrup H; International Tuberous Sclerosis Complex Consensus Group. Tuberous sclerosis complex surveillance and management: recommendations of the 2012 International Tuberous Sclerosis Complex Consensus Conference. Pediatr Neurol 2013;49(4):255-265

60 Moeller JJ, Rahey SR, Sadler RM. Lamotrigine-valproic acid combination therapy for medically refractory epilepsy. Epilepsia 2009; 50(3):475-479

61 Kaminski RM, Matagne A, Patsalos PN, Klitgaard H. Benefit of combination therapy in epilepsy: a review of the preclinical evidence with levetiracetam. Epilepsia 2009;50(3):387-397

62 Lazarowski A, Lubieniecki F, Camarero S, et al. Multidrug resistance proteins in tuberous sclerosis and refractory epilepsy. Pediatr Neurol 2004;30(2):102-106

63 Romanelli P, Najjar S, Weiner HL, Devinsky O. Epilepsy surgery in tuberous sclerosis: multistage procedures with bilateral or multilobar foci. J Child Neurol 2002;17(9):689-692

64 Arya R, Tenney JR, Horn PS, et al. Long-term outcomes of resective epilepsy surgery after invasive presurgical evaluation in children with tuberous sclerosis complex and bilateral multiple lesions. J Neurosurg Pediatr 2015;15(1):26-33

65 Kawai K, Shimizu H, Yagishita A, Maehara T, Tamagawa K. Clinical outcomes after corpus callosotomy in patients with bihemispheric malformations of cortical development. J Neurosurg 2004; 101(1, Suppl)7-15
66 Connolly MB, Hendson G, Steinbok P. Tuberous sclerosis complex: a review of the management of epilepsy with emphasis on surgical aspects. Childs Nerv Syst 2006;22(8):896-908

67 Henry TR. Therapeutic mechanisms of vagus nerve stimulation. Neurology 2002;59(6, Suppl 4):S3-S14

68 Morris GL III, Mueller WM. Long-term treatment with vagus nerve stimulation in patients with refractory epilepsy. The Vagus Nerve Stimulation Study Group E01-E05. Neurology 1999;53(8): 1731-1735

69 Major P, Thiele EA. Vagus nerve stimulation for intractable epilepsy in tuberous sclerosis complex. Epilepsy Behav 2008;13(2): 357-360

70 Parain D, Penniello MJ, Berquen P, Delangre T, Billard C, Murphy JV Vagal nerve stimulation in tuberous sclerosis complex patients. Pediatr Neurol 2001;25(3):213-216

71 Danial NN, Hartman AL, Stafstrom CE, Thio LL. How does the ketogenic diet work? Four potential mechanisms. J Child Neurol 2013;28(8):1027-1033

72 McDaniel SS, Rensing NR, Thio LL, Yamada KA, Wong M. The ketogenic diet inhibits the mammalian target of rapamycin (mTOR) pathway. Epilepsia 2011;52(3):e7-e11

73 Levy RG, Cooper PN, Giri P. Ketogenic diet and other dietary treatments for epilepsy. Cochrane Database Syst Rev 2012;3: CD001903

74 Meikle L, Talos DM, Onda H, et al. A mouse model of tuberous sclerosis: neuronal loss of Tsc1 causes dysplastic and ectopic neurons, reduced myelination, seizure activity, and limited survival. J Neurosci 2007;27(21):5546-5558

75 Feliciano DM, Su T, Lopez J, Platel JC, Bordey A. Single-cell Tsc1 knockout during corticogenesis generates tuber-like lesions and reduces seizure threshold in mice. J Clin Invest 2011;121(4): 1596-1607

76 Meikle L, Pollizzi K, Egnor A, et al. Response of a neuronal model of tuberous sclerosis to mammalian target of rapamycin (mTOR) inhibitors: effects on mTORC1 and Akt signaling lead to improved survival and function. J Neurosci 2008;28(21):5422-5432

77 Talos DM, Kwiatkowski DJ, Cordero K, Black PM, Jensen FE. Cellspecific alterations of glutamate receptor expression in tuberous sclerosis complex cortical tubers. Ann Neurol 2008;63(4): 454-465

78 Curatolo P. Mechanistic target of rapamycin (mTOR) in tuberous sclerosis complex-associated epilepsy. Pediatr Neurol 2015;52(3): 281-289

79 Krueger DA, Wilfong AA, Holland-Bouley K, et al. Everolimus treatment of refractory epilepsy in tuberous sclerosis complex. Ann Neurol 2013;74(5):679-687

80 Orlova KA, Parker WE, Heuer GG, et al. STRADalpha deficiency results in aberrant mTORC1 signaling during corticogenesis in humans and mice. J Clin Invest 2010;120(5):1591-1602

81 Wong M. Mechanisms of epileptogenesis in tuberous sclerosis complex and related malformations of cortical development with abnormal glioneuronal proliferation. Epilepsia 2008;49(1):8-21

82 Crino PB. mTOR: A pathogenic signaling pathway in developmental brain malformations. Trends Mol Med 2011;17(12):734-742

83 Sarnat H, Flores-Sarnat L, Crino P, Hader W, Bello-Espinosa L. Hemimegalencephaly: foetal tauopathy with mTOR hyperactivation and neuronal lipidosis. Folia Neuropathol 2012;50(4): 330-345 